Review Article

\title{
Contrast-Enhanced Ultrasound in the Diagnosis of Hepatocellular Carcinoma and Intrahepatic Cholangiocarcinoma: Controversy over the ASSLD Guideline
}

\author{
Le-Hang Guo and Hui-Xiong Xu \\ Department of Medical Ultrasound, Shanghai Tenth People's Hospital, Tongji University School of Medicine, Shanghai 200072, China \\ Correspondence should be addressed to Hui-Xiong Xu; xuhuixiong@hotmail.com
}

Received 8 August 2014; Revised 13 January 2015; Accepted 13 January 2015

Academic Editor: Ayache Bouakaz

Copyright (C) 2015 L.-H. Guo and H.-X. Xu. This is an open access article distributed under the Creative Commons Attribution License, which permits unrestricted use, distribution, and reproduction in any medium, provided the original work is properly cited.

\begin{abstract}
Hepatocellular carcinoma (HCC) and intrahepatic cholangiocarcinoma (ICC) are both regarded as primary liver cancers, having different biological behaviors and prognoses. Correct differentiation between them is essential for surgical planning and prognosis assessment. In 2005, the American Association for the Study of Liver Diseases (AASLD) recommended that noninvasive diagnosis of HCC is achievable by a single dynamic technique (including contrast-enhanced ultrasound (CEUS)) showing intense arterial uptake followed by washout of contrast in the venous-delayed phases. However, CEUS has been dropped from the diagnostic techniques in the latest AASLD guideline according to the opinion of some authors from Europe that CEUS may offer false positive HCC diagnosis in patients with ICC. Since the update of AASLD guideline has been released, increased attention has been paid to this interesting topic. Remarkable controversy over this issue is present and this removal was not well received in Europe and Asia. This commentary summarized the opinions for the role of CUES in differentiation between HCC and ICC in recent years. It is concluded that prospective studies with strict design and large case series are mandatory to solve the controversies and stratification of ICC in terms of tumor size and liver background is also essential.
\end{abstract}

\section{Background}

Hepatocellular carcinoma (HCC) is the sixth most common neoplasm worldwide. Its incidence has increased in recent years and it is currently the leading cause of death in patients with cirrhosis. Intrahepatic cholangiocarcinoma (ICC) is a rare liver tumor, and its incidence rate has also increased in recent years. Although both ICC and HCC are regarded as primary liver cancers, they have different biological behaviors and prognoses. Correct differentiation between them is essential for surgical planning and prognosis assessment [1-3]. In the recent decade, contrast-enhanced ultrasound (CEUS) has been introduced into clinical practice for diagnosis of focal liver lesion (FLL). Numerous prospective studies including several multicenter studies with large case series have already confirmed the value of CEUS in diagnosis of FLL, and it is widely accepted that CEUS is at least comparable with contrast-enhanced computed tomography (CECT) and contrast-enhanced magnetic resonance imaging (CEMRI) in characterization and detection of FLLs [4-7].

\section{CEUS for ICC}

2.1. Ultrasound and CEUS. Ultrasound (US) has been regarded as the first line imaging modality for detection of FLL, whereas it is difficult for baseline US to distinguish between them because ICC has no specific features $[2,3,8$ 10]. CEUS, with the use of a low acoustic power contrastspecific mode and a gas-filled microbubble contrast agent, allows visualization of macro- and microcirculation of a targeted organ or lesion continuously. The CEUS vascular phases are usually classified into arterial (8-30 s from contrast agent injection), portal (31-120s), and late (121-360 s) phases $[2-5,10,11]$. The CEUS enhancement patterns of 50 massforming ICCs were retrospectively analyzed by Chen et al. [2] and were compared with 50 HCCs. In their study, ICC 
typically produced a peripheral rim-like enhancement in the arterial phase of CEUS, which accounted for $50 \%$ of ICCs. The other enhancement patterns were heterogeneous hypoenhancement (24\%), heterogeneous hyperenhancement $(20 \%)$, and homogeneous hyperenhancement (6\%). However, for HCC, heterogeneous (58\%) and homogeneous (38\%) hyperenhancement were the most common findings in the arterial phase; rim-like hyperenhancement only accounted for $4 \%$ of HCCs. In the late phase, all 50 ICCs and 50 HCCs were hypoenhanced. Time-intensity curve (TIC) demonstrated that the intensities of the peripheral and central portions of the ICCs were lower than those of HCCs. In addition, ICCs showed washout more quickly than HCCs in portal and late phases. The diagnostic performance of both readers in terms of the area under the receiver operating characteristic curve (AUROC) ( 0.745 versus 0.933 for reader 1 , and 0.803 versus 0.911 for reader 2 ), sensitivity ( $28 \%$ versus $90 \%$, and $44 \%$ versus $82 \%$ ), and accuracy (64\% versus $90 \%$, and $71 \%$ versus 90\%) improved significantly after CEUS (all $P<0.05$ ). The interobserver agreement increased from kappa value $(\kappa)=0.575$ at BUS to $\kappa=0.720$ after CEUS. Therefore, CEUS increases the diagnostic performance in differentiation between ICC and HCC significantly, in comparison with conventional ultrasound.

Similar results have been found in the related studies and the typical CEUS manifestations for HCC and ICC are summarized as follows [3-10].

\subsection{1. $\mathrm{HCC}$}

(i) Hyperenhancement in the arterial phase is usually homogeneous and intense but may be inhomogeneous in larger nodules $(>5 \mathrm{~cm})$.

(ii) Washout in late phase is observed in about half of HCC, but more rarely in small nodules $(20-30 \%$ in those $1-2 \mathrm{~cm}, 40-60 \%$ in those $2-3 \mathrm{~cm}$ ).

(iii) The median time of onset of washout was reported to be $2 \mathrm{~min}$ and it is longer in well differentiated HCC than in poor differentiated HCC.

\subsubsection{ICC}

(i) ICCs have a variety of patterns in the arterial phase with the majority showing peripheral rim-like hyperenhancement, while all show washout in the late phase, in contrast to the late enhancement on CECT or CEMRI.

(ii) Early washout at CEUS (usually before $60 \mathrm{~s}$ ).

Intraductal-growing ICC is another type of ICC, which often shows homogeneous hyperenhancement in the arterial phase and washout in the late phase. This type is easy to be differentiated from HCC since it is always located in dilated bile duct whereas HCC is seldom associated with bile duct dilation. Periductal infiltration type of ICC is hard to be detected before surgery; it is also a rare type ICC [10].
2.2. Multimodality Comparison. The vascular structures in the solid tumor are always disorganized and leaky [1214]. The vascular permeability in turn drives tumor-induced angiogenesis and blood flow disturbances, which also facilitates the CT and MRI contrast agents diffusing over time from hyperpermeable intratumoral blood vessels to the interstitium of the tumor, whereas the ultrasound contrast agents are blood pool agents with large size and thus remain in the blood vessels $[15,16]$. Direct comparison between them in the vascular phase is possible, whereas the comparison in the late phase is difficult. Persistent enhancement in the late phase is a typical pattern for ICC on CT and MRI, whereas nearly $100 \%$ ICCs show washout in late phase on CEUS [11, 17]. Chen et al. [11] carried out a comparison study between CEUS and CECT in diagnosis of ICC. The enhancement patterns of ICC on CEUS are consistent with those on CECT in the arterial phase, indicating that it is pathology, not the adopted imaging technology, which determines the enhancement pattern. In the portal phase, ICC fades out more obviously on CEUS than on CECT. In the late phase, all ICCs show washout on CEUS whereas sustained enhancement on CECT. CEUS (accuracy, $80 \%$ ) has the same accuracy as CECT (accuracy, 67.5\%) in diagnosing ICCs and can be used as a new modality for the characterization of ICC.

2.3. ICC: Pathological Correlation with CEUS Pattern. As mentioned above, ICC has different pathological subtypes and the CEUS enhancement pattern is varying. It was confirmed that CT and/or MRI findings of ICC were correlated with pathological components. The hyperenhancing areas always indicated a large number of tumor cells and the delayed enhancement corresponded to fibrotic stroma at pathological examination. Xu et al. [10] found that the hyperenhancing areas on CEUS corresponded to more tumour cells for mass-forming ICCs. There are scarce tumour cells in the centre portion of the tumour and fibrosis is prominent. Microscopically, scarce tumour cells are present in both the peripheral portion and the centre portion. In periductalinfiltrating ICC, fibrosis was more commonly found, whereas in intraductal-growing ICCs, abundant tumor cells were found. Therefore, the imaging findings of ICC on CEUS were related to the degree of carcinoma cell proliferation at pathology; hyperenhancing areas in ICC always indicate increased density of tumor cells.

\section{AASLD Guideline Opinion}

In 2005, the American Association for the Study of Liver Diseases (AASLD) endorsed that noninvasive diagnosis of HCC is achievable by a single dynamic technique (including CEUS) showing intense arterial uptake followed by washout of contrast in the venous-delayed phases [1]. Afterwards, CEUS has also been introduced into other important guidelines and recommendations for the diagnostic work-up of HCC in patients with cirrhosis: the Asian Pacific Association for the Study of the Liver (APASL) consensus recommendations on HCC [18]; the recommendations of the Japanese Society of Hepatology [19]; the European Federation of Societies for 
Ultrasound in Medicine and Biology (EFSUMB) guidelines 2008 [6]; and the World Federation for Ultrasound in Medicine and Biology- (WFUMB-) EFSUMB guidelines 2012 $[4,5]$. Regarding the contrast agent, the APASL guidelines accept the use of Levovist (Schering, Berlin, Germany) or Sonazoid (GE Healthcare, Milwaukee, WI, USA) as contrast agents, the Japanese guidelines accept only the use of Sonazoid, and the WFUMB-EFSUMB guidelines accept both SonoVue (Bracco, Milan, Italy) and Sonazoid as contrast agents. The APASL guidelines recommend the use of either CT or MRI as a first-line technique and resort to CEUS for second/third line in the absence of typical diagnostic $\mathrm{CT}$ and/or MRI patterns, whereas the JSH guidelines accept CEUS even as a first-line option.

However, CEUS has been dropped from the diagnostic techniques in the latest AASLD guideline according to the opinion of some authors from Europe that CEUS may offer false positive HCC diagnosis in patients with ICC. Another reason is that liver CEUS is not approved by Food and Drug Administration (FDA) in the USA. The latter factor indicates that the published results might not be applicable to a North American population. Therefore, the new AASDL guideline has negative opinion on the use of CEUS to make differentiation between HCC and ICC [20].

\section{Current Controversy over AASLD Guideline}

Since the update of AASLD guideline has been released, increased attention has been paid to this interesting topic. Remarkable controversy over this issue is present and this removal was not well received in Europe and Asia. It is questioning whether CEUS should be entirely removed from the important guidelines based on a single, relatively smallscale study that is the source of negative opinion from AASLD [21-28]. Currently, the opinion of negative side was based on limited cases of ICC, and even the limited cases were scattered in different centers, whereas the opinion of positive side was based on the widest ICC/HCC comparison case series. However, subanalysis was absent in both sides.

4.1. Negative Opinion. In 2010, Vilana et al. [21], on behalf of the Barcelona Clinic Liver Cancer (BCLC) group, analyzed the CEUS enhancement patterns of 21 patients with ICCs from 2003 to 2009 and then concluded that CEUS was not able to be used to differentiate ICC and HCC since the enhancement patterns were similar with HCC in 10 cases that they showed homogeneous arterial hyperenhancement followed by washout. They also concluded that CEUS should not be used as the sole imaging technique for conclusive HCC diagnosis. The potential risk of positive diagnosis of HCC in cirrhosis by CEUS should therefore be kept in high consideration.

In 2012, Bohle et al. [23] retrospectively analyzed the CEUS pattern of 39 patients with HCC, 11 patients with ICCs, 3 patients with Klatskin tumors, and 4 patients with gallbladder carcinomas; they found that HCC and ICC differ to some extent in their CEUS enhancement pattern. Incomplete arterial hyperenhancement is more often seen in ICC. A rim sign seems to be specific for ICC but is only rarely present. Due to overlapping characteristics, a reliable differentiation between the two tumor types by CEUS alone is very often not possible.

4.2. Equivocal Opinion. In 2013, Galassi et al. [24] analyzed the CEUS patterns of 25 small ICCs from three Italian centers between 2003 and 2011, which means less than one case in each center per year. They found that CEUS was at risk of misdiagnosis of ICC for HCC in a significantly higher number of cases than in CT (52\% versus $4.2 \%)$ and MRI (52\% versus 9.1\%). In the arterial phase, ICC lacked global hyperenhancement in approximately $50 \%$ of cases at CEUS and the degree of intensity of washout in the late phase was marked in $24 \%$ of nodules. They concluded that CEUS misdiagnosed as HCC a significantly higher number of ICC lesions in cirrhotic patients than CT and MRI. However, some CEUS contrast features can help suspect ICC, especially in some cases with inconclusive CT or MRI.

4.3. Positive Opinion. In 2012, Barreiros et al. [25] firstly commented on the AASLD guideline regarding removal of CEUS for diagnosis of HCC. In the opinion of the authors, CEUS should be part not only of most, but all international HCC guidelines. Giorgio et al. [26] made a comment on the study of Galassi et al. [24] and concluded it was an exaggerated fear for misdiagnosis of ICC in cirrhosis at CEUS. They endorsed the study of Chen et al. [2] that it was still the widest ICC/HCC comparison case series, even if not specifically devoted to ICC on cirrhosis, CEUS significantly improved the diagnostic performance in the differentiation between ICC and HCC.

In 2013, Korean Association for the Study of CEUS also commented on this issue. They regarded this removal as controversial and not well received in Europe and Asia [27]. Bohle et al. [23] also commented on this issue and concluded that there was no need to remove CEUS from the AASLD practice guidelines as part of the diagnostic algorithm for HCC in cirrhotic patients. Limited market availability (i.e., liver CEUS is not approved by FDA in the USA) is also not a justification to eliminate a valuable diagnostic tool from the algorithm. CEUS should not be considered as a "second-line" imaging technique in the patient care.

Li et al. [29] firstly evaluated the usefulness of CEUS in differentiating ICC from HCC in cirrhotic patients. They found that analysis of detailed temporal enhancement features on CEUS is helpful to differentiate ICC from HCC in cirrhosis. If a nodule in cirrhotic liver displays hyperenhancement in the arterial phase followed by early and marked washout in the portal phase, the nodule is highly suspicious of ICC rather than HCC since, in comparison with ICC, the washout in portal phase is moderate and sometimes even shows iso-enhancing for HCC. Their results provided the latest evidence to rebuke the opinion from AASLD.

\section{Discussion and Conclusion}

The controversy over AASLD guideline regarding the differentiation between HCC and ICC using CEUS continues. 
When reanalyzing the data of Vilana et al. [21], we found that $52.4 \%$ of their cases showed periphery hyperenhancement in arterial phase, $71.4 \%$ showed hypoenhancement in portal phase, and $100 \%$ showed hypoenhancement in late phase. All of the features are characteristic for ICC instead of HCC. In addition, no HCC was included and no comparison study was performed; thus no data supported the conclusion directly. The characteristic pattern of periphery hyperenhancement for ICC in arterial phase was overlooked, as well as quick washout in portal and late phases. The low incidence of ICC was also overlooked; in an analysis of 993 adult cirrhotic liver explants with liver cancer, ICC was only found in $1 \%$ of all the cases [22]. The study of BCLC group was carried out in a long period and only 3 cases per year were evaluated, which aroused doubt about their experience and also indicated that the risk for misdiagnosis is only less than 3/year [21]. The low risk for misdiagnosis could also happen for CECT or CEMRI whereas they were not removed from the diagnostic workup. Additionally, the CEUS pattern for ICC would suggest a diagnosis of malignancy with almost $100 \%$ specificity since nearly $100 \%$ showed washout in late phase, whereas the pattern of CT or MRI might not be specific for malignancy (washin followed by sustained enhancement) that some had been misdiagnosed as hemangioma or abscess. This is an important point that biopsy might not be feasible in all the patients. In addition, it should be pointed out that the study was not a prospective and controlled study design. Finally, to overcome the uncertainty in relation to the complex visual investigation of dynamic loops at CEUS, quantification of CEUS is necessary, which may facilitate differential diagnosis between ICC and HCC $[30,31]$. Regarding the study of Bohle et al. [23], the conclusion was obviously paradox and was not based on the results. In addition, no diagnostic test was performed to differentiate HCC and ICC.

Barreiros et al. [25] refuted the opinion of AASLD with the following points. Firstly, CEUS has been introduced more than 10 years for liver imaging in many European and Asian countries, but FDA approval in the US is still lacking. Thus the evidence for the AASLD opinion is scarce. Secondly, ICC is a rare tumour in liver cirrhosis (about $1-3 \%$ of newly developed tumors); thus it should not overestimate the risk for misdiagnosis. Thirdly, when referring the study of Vilana et al. [21], it was a nonprospective and noncontrolled study design. Therefore, the quality of the study and the clinical consequences of this possible risk do not seem to justify the complete removal of CEUS from the imaging armamentarium. In addition, the study was carried out without enough experience; the investigators were not well trained, and CEUS was not performed in expert hand.

Therefore, the evidence for positive opinion is accumulating. Prospective studies with strict design are mandatory to solve the controversies. Future studies should focus on the imaging features of ICC in healthy livers and cirrhotic livers. Prospective case control studies with large case series are needed. Stratification of ICC in terms of tumor size and liver background is also essential. Finally, CEUS has the reason to retain in the guidelines because there is no radiation exposure to the patient, negligible nephrotoxicity, and a low risk of allergic and hypersensitivity reactions. CEUS is also applicable when CT or MRI is contraindicated.

\section{Conflict of Interests}

The authors declare that there is no conflict of interests regarding the publication of this paper.

\section{Authors' Contribution}

Our paper has been reviewed and approved by all authors and all authors have made significant contribution to the content of this paper.

\section{Acknowledgments}

This work was supported in part by Grants 20114003 and 2013SY066 from Shanghai Municipal Commission of Health and Family Planning, Grant 14441900900 from Science and Technology Commission of Shanghai Municipality, Grants 81301229, 81301299, and 81371570 from the National Natural Science Foundation of China, and Grant 2012045 from Shanghai Municipal Human Resources and Social Security Bureau.

\section{References}

[1] J. Bruix and M. Sherman, "Management of hepatocellular carcinoma," Hepatology, vol. 42, no. 5, pp. 1208-1236, 2005.

[2] L.-D. Chen, H.-X. Xu, X.-Y. Xie et al., "Intrahepatic cholangiocarcinoma and hepatocellular carcinoma: differential diagnosis with contrast-enhanced ultrasound," European Radiology, vol. 20, no. 3, pp. 743-753, 2010.

[3] R. Li, X. Zhang, K.-S. Ma et al., "Dynamic enhancing vascular pattern of intrahepatic peripheral cholangiocarcinoma on contrast-enhanced ultrasound: the influence of chronic hepatitis and cirrhosis," Abdominal Imaging, vol. 38, no. 1, pp. 112-119, 2013.

[4] M. Claudon, C. F. Dietrich, B. I. Choi et al., "Guidelines and good clinical practice recommendations for contrast enhanced ultrasound (CEUS) in the liver-update 2012: a WFUMBEFSUMB initiative in cooperation with representatives of AFSUMB, AIUM, ASUM, FLAUS and ICUS," Ultrasound in Medicine \& Biology, vol. 39, no. 2, pp. 187-210, 2013.

[5] M. Claudon, D. Cosgrove, T. Albrecht et al., "Guidelines and good clinical practice recommendations for contrast enhanced ultrasound (CEUS) - update 2008," Ultraschall in der Medizin, vol. 29, no. 1, pp. 28-44, 2008.

[6] K. Seitz, T. Bernatik, D. Strobel et al., "Contrast-enhanced ultrasound (CEUS) for the characterization of focal liver lesions in clinical practice (DEGUM Multicenter Trial): CEUS vs. MRI-a prospective comparison in 269 patients," Ultraschall in der Medizin, vol. 31, no. 5, pp. 492-499, 2010.

[7] K. Seitz, D. Strobel, T. Bernatik et al., "Contrast-Enhanced ultrasound (CEUS) for the characterization of focal liver lesions-prospective comparison in clinical practice: CEUS vs. CT (DEGUM Multicenter Trial) parts of this manuscript were presented at the Ultrasound Dreiländertreffen 2008, Davos," Ultraschall in der Medizin, vol. 30, no. 4, pp. 383-389, 2009. 
[8] C. Li, W. Wang, H. Ding et al., "Value of contrast-enhanced sonography in the diagnosis of peripheral intrahepatic cholangiocarcinoma," Journal of Clinical Ultrasound, vol. 39, no. 8, pp. 447-453, 2011.

[9] W. T. Kong, W. P. Wang, W. W. Zhang, Y. D. Qiu, H. Ding, and B. J. Huang, "Contribution of contrast-enhanced sonography in the detection of intrahepatic cholangiocarcinoma," Journal of Ultrasound in Medicine, vol. 33, no. 2, pp. 215-220, 2014.

[10] H.-X. Xu, L.-D. Chen, L.-N. Liu, Y.-F. Zhang, L.-H. Guo, and C. Liu, "Contrast-enhanced ultrasound of intrahepatic cholangiocarcinoma: correlation with pathological examination," British Journal of Radiology, vol. 85, no. 1016, pp. 1029-1037, 2012.

[11] L.-D. Chen, H.-X. Xu, X.-Y. Xie et al., "Enhancement patterns of intrahepatic cholangiocarcinoma: comparison between contrast-enhanced ultrasound and contrast-enhanced CT," British Journal of Radiology, vol. 81, no. 971, pp. 881-889, 2008.

[12] R. E. Carraway and D. E. Cochrane, "Enhanced vascular permeability is hypothesized to promote inflammation-induced carcinogenesis and tumor development via extravasation of large molecular proteins into the tissue," Medical Hypotheses, vol. 78, no. 6, pp. 738-743, 2012.

[13] J. A. Nagy, A. M. Dvorak, and H. F. Dvorak, "Vascular hyperpermeability, angiogenesis, and stroma generation," Cold Spring Harbor Perspectives in Medicine, vol. 2, no. 2, Article ID a006544, 2012.

[14] S. J. Kim, Y. Lee, N. Y. Kim et al., "Pancreatic adenocarcinoma upregulated factor, a novel endothelial activator, promotes angiogenesis and vascular permeability," Oncogene, vol. 32, no. 31, pp. 3638-3647, 2013.

[15] M. A. Rosen and M. D. Schnall, "Dynamic contrast-enhanced magnetic resonance imaging for assessing tumor vascularity and vascular effects of targeted therapies in renal cell carcinoma," Clinical Cancer Research, vol. 13, no. 2, pp. 770s-776s, 2007.

[16] C. A. Cuenod, L. Fournier, D. Balvay, and J.-M. Guinebretière, "Tumor angiogenesis: pathophysiology and implications for contrast-enhanced MRI and CT assessment," Abdominal Imaging, vol. 31, no. 2, pp. 188-193, 2006.

[17] M. D’Onofrio, F. Vecchiato, V. Cantisani et al., "Intrahepatic peripheral cholangiocarcinoma (IPCC): comparison between perfusion ultrasound and CT imaging," Radiologia Medica, vol. 113 , no. 1, pp. 76-86, 2008.

[18] M. Omata, L. A. Lesmana, R. Tateishi et al., "Asian pacific association for the study of the liver consensus recommendations on hepatocellular carcinoma," Hepatology International, vol. 4, no. 2, pp. 439-474, 2010.

[19] M. Kudo, N. Izumi, N. Kokudo et al., "Management of hepatocellular carcinoma in Japan: consensus-based clinical practice guidelines proposed by the Japan society of hepatology (JSH) 2010 updated version," Digestive Diseases, vol. 29, no. 3, pp. 339364, 2011.

[20] J. Bruix and M. Sherman, "Management of hepatocellular carcinoma: an update," Hepatology, vol. 53, no. 3, pp. 1020-1022, 2011.

[21] R. Vilana, A. Forner, L. Bianchi et al., "Intrahepatic peripheral cholangiocarcinoma in cirrhosis patients may display a vascular pattern similar to hepatocellular carcinoma on contrastenhanced ultrasound," Hepatology, vol. 51, no. 6, pp. 2020-2029, 2010.

[22] M. Vallin, N. Sturm, G. Lamblin et al., "Unrecognized intrahepatic cholangiocarcinoma: an analysis of 993 adult cirrhotic liver explants," Clinical Transplantation, vol. 27, no. 3, pp. 403-409, 2013.

[23] W. Bohle, P. U. Clemens, T. Heubach, and W. G. Zoller, "Contrast-enhanced ultrasound (CEUS) for differentiating between hepatocellular and cholangiocellular carcinoma," Ultraschall in der Medizin, vol. 33, no. 7, pp. E191-E195, 2012.

[24] M. Galassi, M. Iavarone, S. Rossi et al., "Patterns of appearance and risk of misdiagnosis of intrahepatic cholangiocarcinoma in cirrhosis at contrast enhanced ultrasound," Liver International, vol. 33, no. 5, pp. 771-779, 2013.

[25] A. P. Barreiros, F. Piscaglia, and C. F. Dietrich, "Contrast enhanced ultrasound for the diagnosis of hepatocellular carcinoma (HCC): comments on AASLD guidelines," Journal of Hepatology, vol. 57, no. 4, pp. 930-932, 2012.

[26] A. Giorgio, L. Montesarchio, and V. Giorgio, "Misdiagnosis of intrahepatic cholangiocarcinoma in cirrhosis at contrastenhanced ultrasound: a real new clinical entity or just an exaggerated fear?" Liver International, vol. 33, no. 10, p. 1608, 2013.

[27] J. Y. Jang, M. Y. Kim, S. W. Jeong et al., "Current consensus and guidelines of contrast enhanced ultrasound for the characterization of focal liver lesions," Clinical and Molecular Hepatology, vol. 19, no. 1, pp. 1-16, 2013.

[28] C. F. Dietrich, X. W. Cui, B. Boozari, M. Hocke, and A. Ignee, "Contrast-enhanced ultrasound (CEUS) in the diagnostic algorithm of hepatocellular and cholangiocellular carcinoma, comments on the AASLD guidelines," Ultraschall in der Medizin, vol. 33, no. 1, pp. S57-S66, 2012.

[29] R. Li, M. X. Yuan, K. S. Ma et al., "Detailed analysis of temporal features on contrast enhanced ultrasound may help differentiate intrahepatic cholangiocarcinoma from hepatocellular carcinoma in cirrhosis," PLoS ONE, vol. 9, no. 5, Article ID e98612, 2014.

[30] J. M. Hudson, R. Karshafian, and P. N. Burns, "Quantification of flow using ultrasound and microbubbles: a disruption replenishment model based on physical principles," Ultrasound in Medicine and Biology, vol. 35, no. 12, pp. 2007-2020, 2009.

[31] E. Amarteifio, S. Wormsbecher, M. Krix et al., "Dynamic contrast-enhanced ultrasound and transient arterial occlusion for quantification of arterial perfusion reserve in peripheral arterial disease," European Journal of Radiology, vol. 81, no. 11, pp. 3332-3338, 2012. 


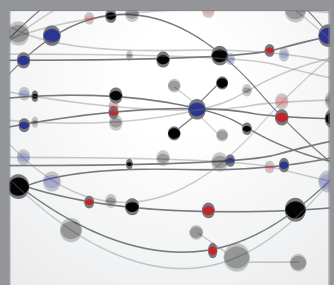

The Scientific World Journal
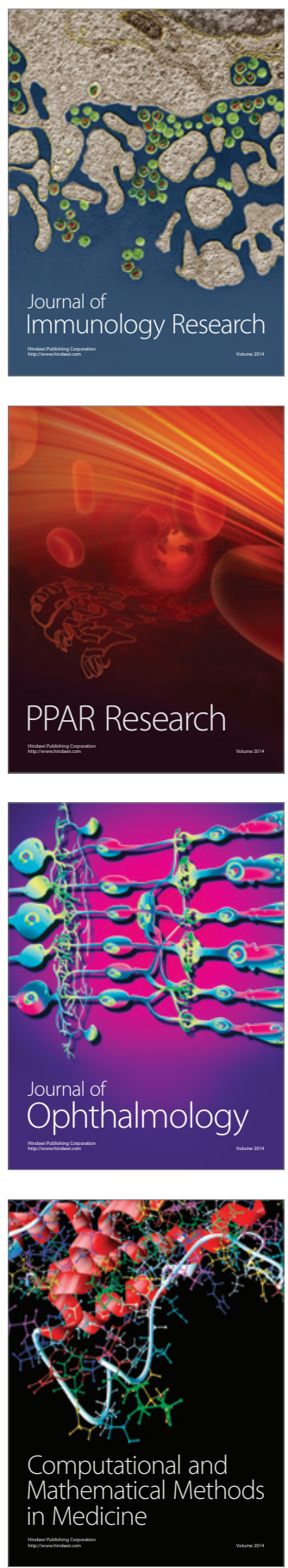

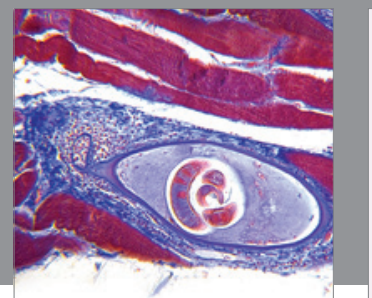

Gastroenterology

Research and Practice
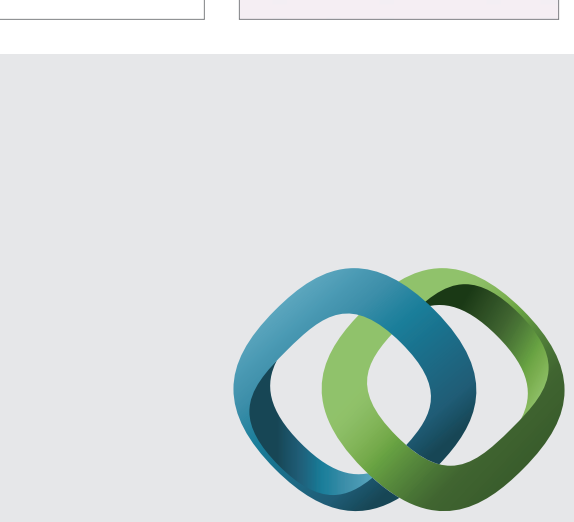

\section{Hindawi}

Submit your manuscripts at

http://www.hindawi.com
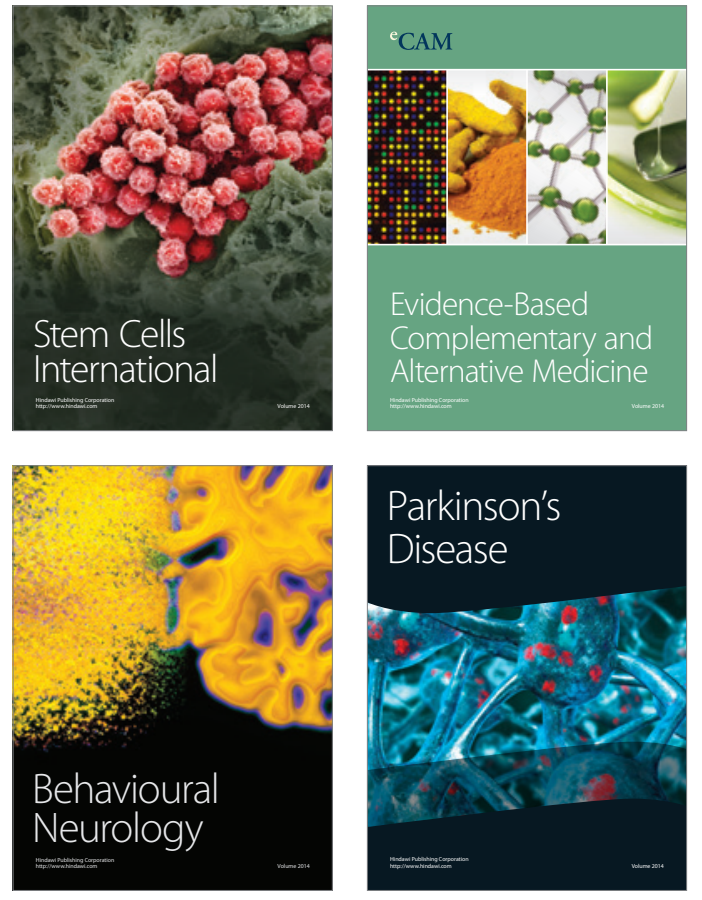
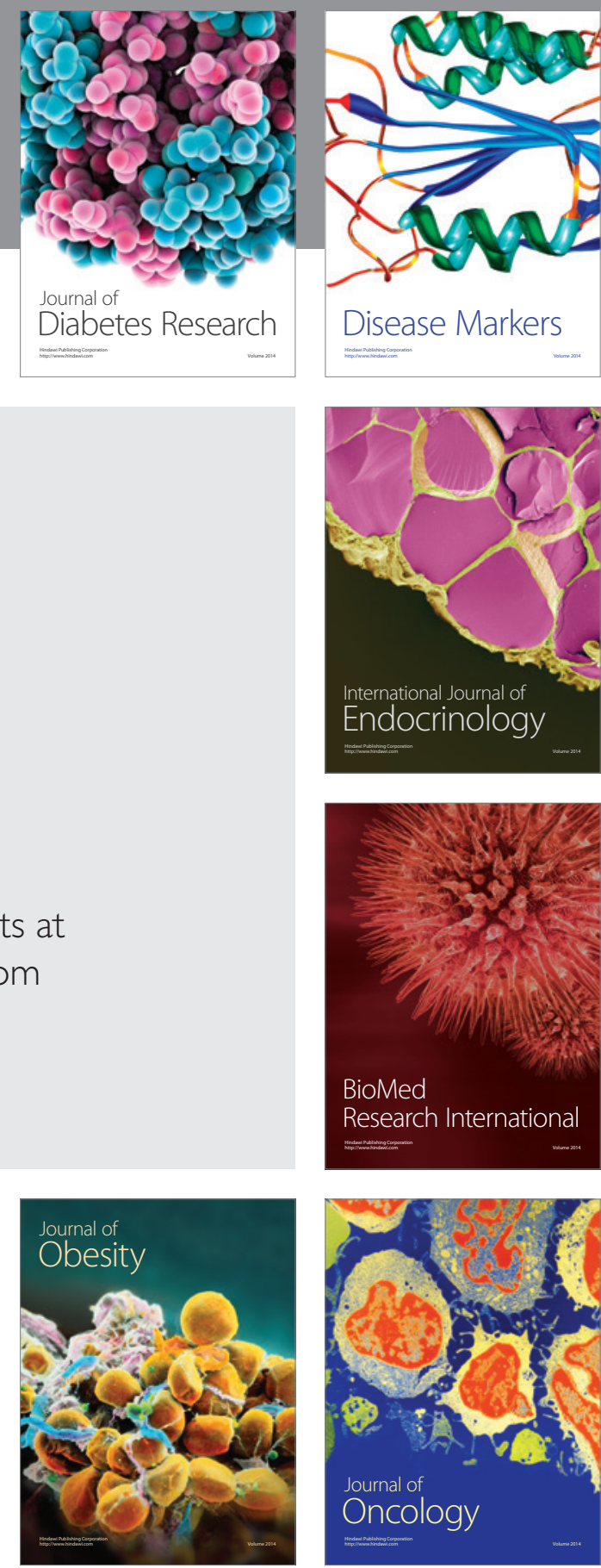

Disease Markers
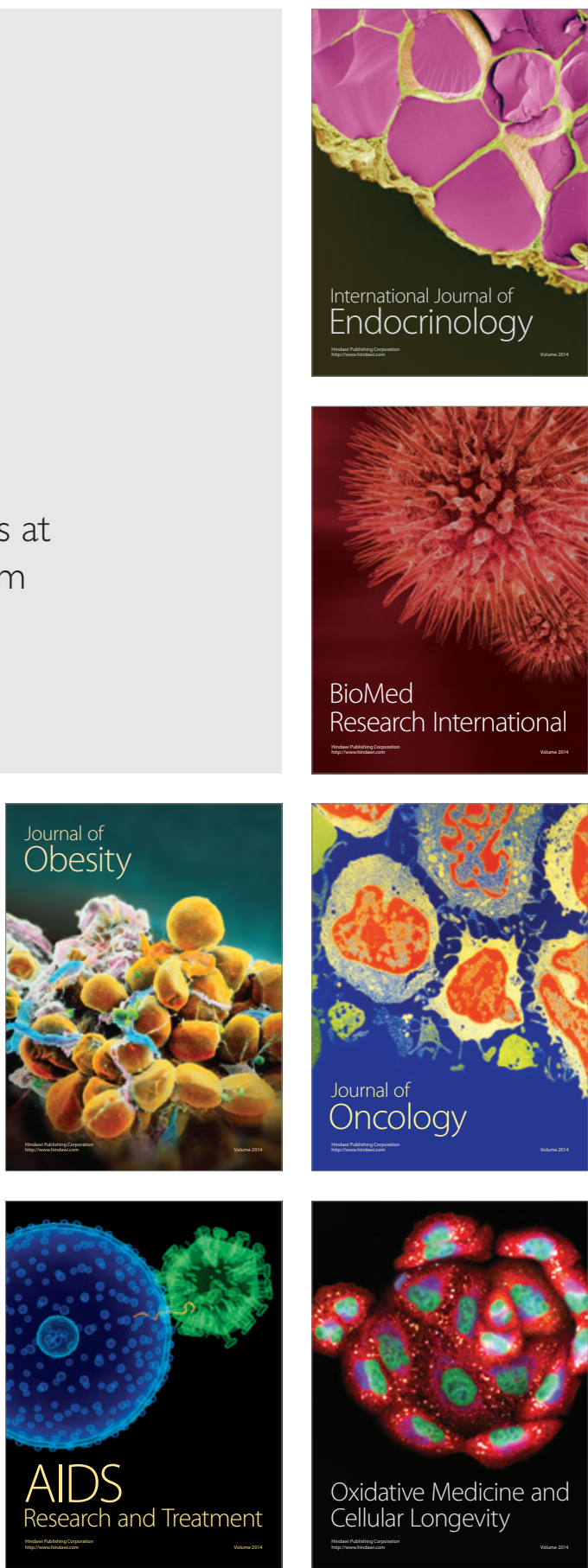